\title{
The Structural Basis for Calcium Inhibition of Lipid Kinase PI4K IIa and Comparison With the Apo State
}

\author{
A. BAUMLOVA ${ }^{1}$, J. GREGOR $^{1}$, E. BOURA $^{1}$ \\ ${ }^{1}$ Institute of Organic Chemistry and Biochemistry, Czech Academy of Sciences, Prague, Czech \\ Republic
}

Received March 9, 2016

Accepted May 13, 2016

On-line August 19, 2016

\begin{abstract}
Summary
PI4K IIa is a critical enzyme for the maintenance of Golgi and is also known to function in the synaptic vesicles. The product of its catalytical function, phosphatidylinositol 4-phosphate (PI4P), is an important lipid molecule because it is a hallmark of the Golgi and TGN, is directly recognized by many proteins and also serves as a precursor molecule for synthesis of higher phosphoinositides. Here, we report crystal structures of PI4K IIa enzyme in the apo-state and inhibited by calcium. The apostructure reveals a surprising rigidity of the active site residues important for catalytic activity. The structure of calcium inhibited kinase reveals how calcium locks ATP in the active site.
\end{abstract}

\section{Key words}

Lipid kinase • Calcium • Phosphatidylinositol • Crystal structure

\section{Corresponding author}

E. Boura, Institute of Organic Chemistry and Biochemistry, Czech Academy of Sciences, Flemingovo nám. 2, 16610 Prague 6, Czech Republic. E-mail: boura@uochb.cas.cz

\section{Introduction}

Phosphaditylinositol 4-phosphate (PI4P) is an important regulatory molecule that helps to define Golgi and trans-Golgi network (TGN) and serves as a precursor for higher phosphoinositides (Cauvin and Echard 2015, Tan and Brill 2014). Phosphoinositides also serve as specific markers for intracellular membranes that are more or less specifically recognized by phosphoinositide binding domains such as $\mathrm{PH}$ domains (Hammond and Balla 2015, Boura and Hurley 2012). In humans, PI4P is synthesized by four phosphatidylinositol 4-kinases (PI4Ks) divided into two groups. We have two type II PI4Ks (PI4K II $\alpha$ and PI4K II $\beta$, also known as PI4K2A and PI4K2B) and two type III PI4Ks (PI4K III $\alpha$ and PI4K III $\beta$ also known as PI4KA and PI4KB) (Boura and Nencka 2015, Clayton et al. 2013). Type II PI4Ks are inhibited by calcium whereas type III kinases are sensitive to wortmannin, an inhibitor of PI3 kinases (Downing et al. 1996). The Golgi localized PI4K II $\alpha$ is responsible for the synthesis of $\sim 50 \%$ of total PI4P and together with PI4K III $\beta$ is important for Golgi related trafficking (Jovic et al. 2012, Jovic et al. 2014). Notably, some lipids important in Parkinson's or Gaucher's disease such as ceramide or glucocylceramide are transported in PI4P dependent fashion (Waugh 2015, Dornan et al. 2016).

Until recently no structures of PI4Ks were available at all. It was only in the last few years that structural knowledge of PI4K biology began to emerge. The structures of PI4K II $\alpha$ and II $\beta$ were solved in a nucleotide-bound complex (Baumlova et al. 2014, Zhou et al. 2014, Klima et al. 2015) and the structure of PI4K III $\beta$ was solved in complex with the small GTPase Rab11 and an unspecific lipid kinase inhibitor Pik93 (Burke et al. 2014). The structural insight confirmed bioinformatics-based predictions (Brown and Auger 2011) that type II PI4Ks adopt an atypical lipid kinase fold where only the C-lobes bear structural similarity to other lipid kinases and the N-lobes resemble the N-lobes of a protein kinase. On the other hand, structural analysis of PI4K III $\beta$ revealed a typical kinase fold very similar to PI3Ks. Importantly, PI4K III $\beta$ is considered a potential 
drug target against plus-RNA viruses (Altan-Bonnet and Balla 2012). The synthesis of highly specific inhibitors and structural basis of their function was described recently (Mejdrova et al. 2015), as was the structural mechanism of PI4K III $\beta$ recruitment and activation by the Golgi resident ACBD3 protein (Klima et al. 2016).

For most kinases the structure of the apo-state is very difficult to obtain as a ligand stabilizing the kinase domain is critical for successful crystallization experiments. This missing structural information is necessary to better understand co-factor recognition by PI4K II $\alpha$. Therefore, it would also be useful for inhibitor design. Previously, to obtain crystals of PI4K II $\alpha$ with ATP, we had to remove the intrinsically disordered regions that impede crystallization (Rozycki and Boura 2014) and replace a flexible loop that is palmitoylated in cells with T4 lysozyme (Baumlova et al. 2014). Now we took advantage of the available crystals with ATP and we used them for the first round of microseeding using conditions without a nucleotide. After further optimization we obtained diffraction quality crystals of PI4K II $\alpha$ with no ligand bound. In this study we present the structure of PI4K II $\alpha$ in the apo-state. To our surprise, we demonstrate that most of the residues in the active site have the same conformation as when bound to ATP with the exception of the G-loop, a region that binds ATP phosphate groups.

Next we were interested in the mechanism of inhibition by calcium ions. We were able to obtain calcium and ATP containing crystals. Our structural analysis provides atomic insight into calcium ions inhibition of PI4K II $\alpha$.

\section{Materials and Methods}

\section{Protein expression and purification}

The proteins were expressed and purified using previously developed protocols (Boura et al. 2010, Nemecek et al. 2013). Briefly, the proteins were expressed in E. coli strain BL21 Star. After affinity purification on Ni-NTA resin (Machery-Nagel) the solubility tag (6xHis-GB1) was removed by TEV protease and the proteins were further purified using Superdex 200 column (GE Healthcare). Proteins were concentrated to $8 \mathrm{mg} / \mathrm{ml}$ and stored at $-80{ }^{\circ} \mathrm{C}$ until needed.

\section{Kinase luminescence assay}

The commercial ADP-Glo assay (Promega) was used according to previously published protocol (Tai et al. 2011). Briefly, reactions were carried out in a total volume of $5 \mu \mathrm{l}$ to contain $100 \mathrm{nM}$ PI4K II $\alpha$ (wt or mutants), $50 \mu \mathrm{M}$ PI in kinase buffer (20 mM Tris pH 7.5; $5 \mathrm{mM} \mathrm{MgCl}_{2} ; 0.2 \%$ Triton-X; $0.1 \mathrm{mg} / \mathrm{ml} \mathrm{BSA} ; 2 \mathrm{mM}$ DTT) with and without $10 \mathrm{mM}$ calcium chloride. The reaction was carried out for $60 \mathrm{~min}$ at $\mathrm{RT}(295 \mathrm{~K})$ and the amount of hydrolyzed ATP was measured according to the manufacturer's protocol using TECAN infinite M 1000 plate reader.

\section{Crystallographic analysis}

Diffraction quality crystals of the apo-state enzyme were obtained by microseeding into a Morpheus screen (Gorrec 2009) based condition (12.5\% w/v PEG 1000, $12.5 \%$ w/v PEG 3350, $12.5 \%$ v/v MPD, $0.1 \mathrm{M}$ bicine/Trizma base $\mathrm{pH} 8.5$ ). The calcium bound crystals grew in similar conditions supplemented with $30 \mathrm{mM}$ calcium chloride. The complete datasets were collected at the BESSY 14.1 MX beamline using Pilatus $6 \mathrm{M}$ detector (Mueller et al. 2012). Crystals of the apo-state diffracted to $3 \AA$, calcium bound crystals diffracted to $2.4 \AA$ and they both belonged to the $\mathrm{P} 2{ }_{1} 2{ }_{1} 2$ spacegroup. Data were scaled and integrated in XDSAPP (Krug et al. 2012) and were cut at $\mathrm{CC}_{1 / 2}=50 \%$. The structures were solved by molecular replacement using the structure of ATP bound PI4K II $\alpha$ (pdb code 4PLA) as the search model. The structure was refined in Phenix (Adams et al. 2010) and Coot (Emsley et al. 2010) to $\mathrm{R}_{\text {work, apo }}=24.64 \%$ and $\mathrm{R}_{\text {free, apo }}=28.89 \%$ and to $\mathrm{R}_{\text {work, } \mathrm{Ca}}=21.92 \%$ and $\mathrm{R}_{\text {free, } \mathrm{Ca}}=25.77 \%$ and to good stereochemistry as summarized in Table 1.

\section{Results}

The overall conformation of the kinase, as expected, did not change in either case (Fig. 1). The kinase has $\mathrm{N}$ - and $\mathrm{C}$-lobes with the active site in-between. The T4 lysozyme that improved solubility of the recombinant protein and the quality of crystals.

Prior to our study, only structures with a nucleotide or nucleotide analogue MD59 (Dejmek et al. 2015) were available. We compared the apo-active site to the active site bound to ATP (pdb code 4PLA) and to the active site bound to MD59 (pdb code 4YC4). Analysis of the apo-state and ATP bound state (Fig. 2A) revealed surprising rigidity of the active site residues important for catalytic activity (S134, V150, K152, Q261, and I345). 
Table 1. Statistics of crystallographic data collection and refinement.

\begin{tabular}{|c|c|c|}
\hline Crystal & apo PI4K II & Ca + ATP bound PI4K IIa \\
\hline Wavelength (A) & 0.9796 & 0.9834 \\
\hline Resolution range $(\AA)$ & $48.97-2.80(2.89-2.80)$ & $48.95-2.28(2.362-2.28)$ \\
\hline Space group & $\mathrm{P} 2{ }_{1} 2{ }_{1}$ & $\mathrm{P} 2{ }_{1} 2_{1} 2$ \\
\hline Unit-cell parameters $(\AA, \circ)$ & $\mathrm{a}=80.01 \mathrm{~b}=101.52 \mathrm{c}=78.14, \alpha=\beta=\gamma=90$ & $a=78.88 b=102.03 c=78.93, \alpha=\beta=\gamma=90$ \\
\hline Unique reflections & $16220(1522)$ & $29715(1409)$ \\
\hline Multiplicity & 7.07 & $7.3(7.3)$ \\
\hline Completeness (\%) & $99.22(94.89)$ & $99.93(99.97)$ \\
\hline Mean $I / \sigma(I)$ & $8.38(0.66)$ & $11.16(1.46)$ \\
\hline$C C_{1 / 2}^{*}$ & $48 \%$ & $54.2 \%$ \\
\hline Wilson B factor $\left(\AA^{2}\right)$ & 70.5 & 44.9 \\
\hline$R_{\text {merge }}$ & $23.4 \%(198.5 \%)$ & $13.2 \%(136.9 \%)$ \\
\hline Reflections used for $R_{\text {free }}(\%)$ & $5 \%$ & $5 \%$ \\
\hline$R_{\text {work }}$ & $24.64 \%$ & $21.92 \%$ \\
\hline$R_{\text {free }}$ & $28.89 \%$ & $25.77 \%$ \\
\hline No. of non-H atoms & 3894 & 4228 \\
\hline R.m.s.d., bonds $(\AA)$ & 0.004 & 0.009 \\
\hline R.m.s.d., angles $\left({ }^{\circ}\right)$ & 0.91 & 0.99 \\
\hline Ramachandran favored (\%) & 97 & 96 \\
\hline Ramachandran outliers (\%) & 0.21 & 0 \\
\hline
\end{tabular}

* value used for data cut-off.

The whole glycine rich G-loop (130-136) was in a different conformation. However, this is expected as it binds the ATP phosphate groups. Probably the G-loop is highly flexible which is supported by its high B-factor (106.6) compared to the rest of the structure (76.5). The remarkable fact is that even the side chains of residues that do make hydrogen bonds with ATP (K152 and Q261) are in the same conformation as in the apo-structure (Fig. 2A). Comparison of the apo- and the MD59 bound states (Fig. 2B) revealed similar conformations of the G-loop. This is in agreement with the fact that the G-loop binds the phosphates of ATP which are not present in nucleotide analog MD59. The residues that contribute to MD59 binding are in the same conformation as residues that bind the ATP adenine ring and sugar (Klima et al. 2015). However, the conformation of the D346 residue is different. In the apo-state it resembles the conformation it has in the ATP bound state. PI4K II $\alpha$ also contains a lateral pocket within its C-lobe that is capable of ATP binding and its prosed physiological function is to position correctly position the kinase in respect to the lipid bilayer (Baumlova et al. 2014) as its surface exposed tryptophan residues were observed to be inserted to the lipid bilayer (Zhou et al. 2014). However, the comparison of ATP bound and ligand bound lateral pocket did not reveal any conformational change (Fig. 2C).

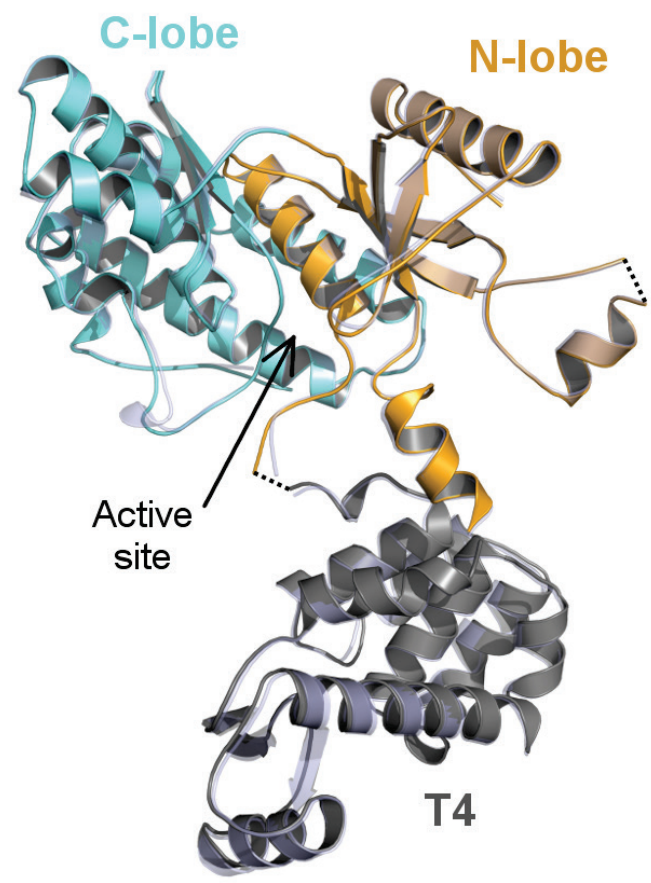

Fig. 1. The overall fold of PI4K IIa. The general fold is the same in the apo and liganded state. C-lobe is colored in cyan, N-lobe in orange and T4 lysozyme in gray. The superimposed liganded kinase ( $p d b$ code 4PLA) is colored in light blue and transparent. 

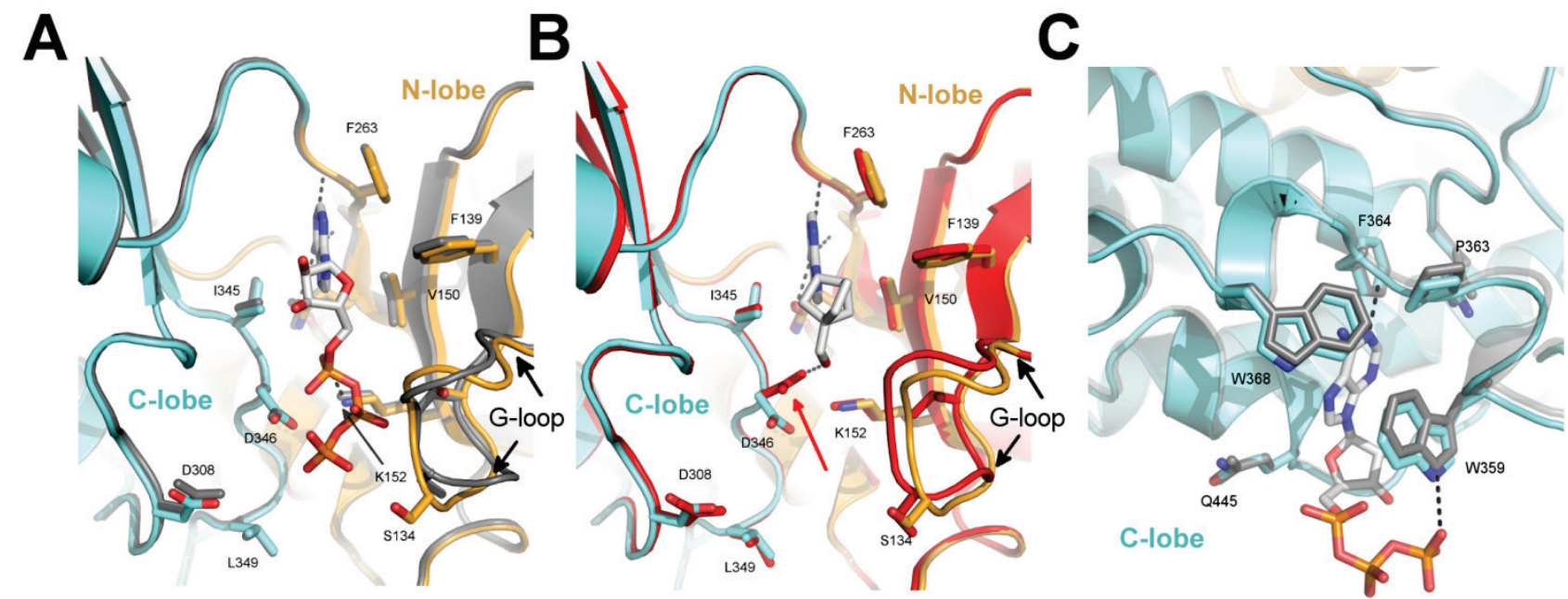

Fig. 2. Comparison of the ATP binding sites. A) The apo and ATP bound active site. C-lobe is colored in cyan, N-lobe in orange and T4 lysozyme in gray. The superimposed ATP bound kinase (pdb code 4PLA) is in gray except for the ATP that is colored according its elements. B) The apo and MD59 bound active sites. C-lobe is colored in cyan, N-lobe in orange and T4 lysozyme in gray. The superimposed ATP bound kinase (pdb code 4YC4) is in red except for the ATP that is colored according its elements. The red arrow points to the flexible D346 residue. C) The apo and ATP bound lateral pocket. C-lobe is colored in cyan. The superimposed ATP bound kinase (pdb code 4PLA) is in gray except for the ATP that is colored according its elements.
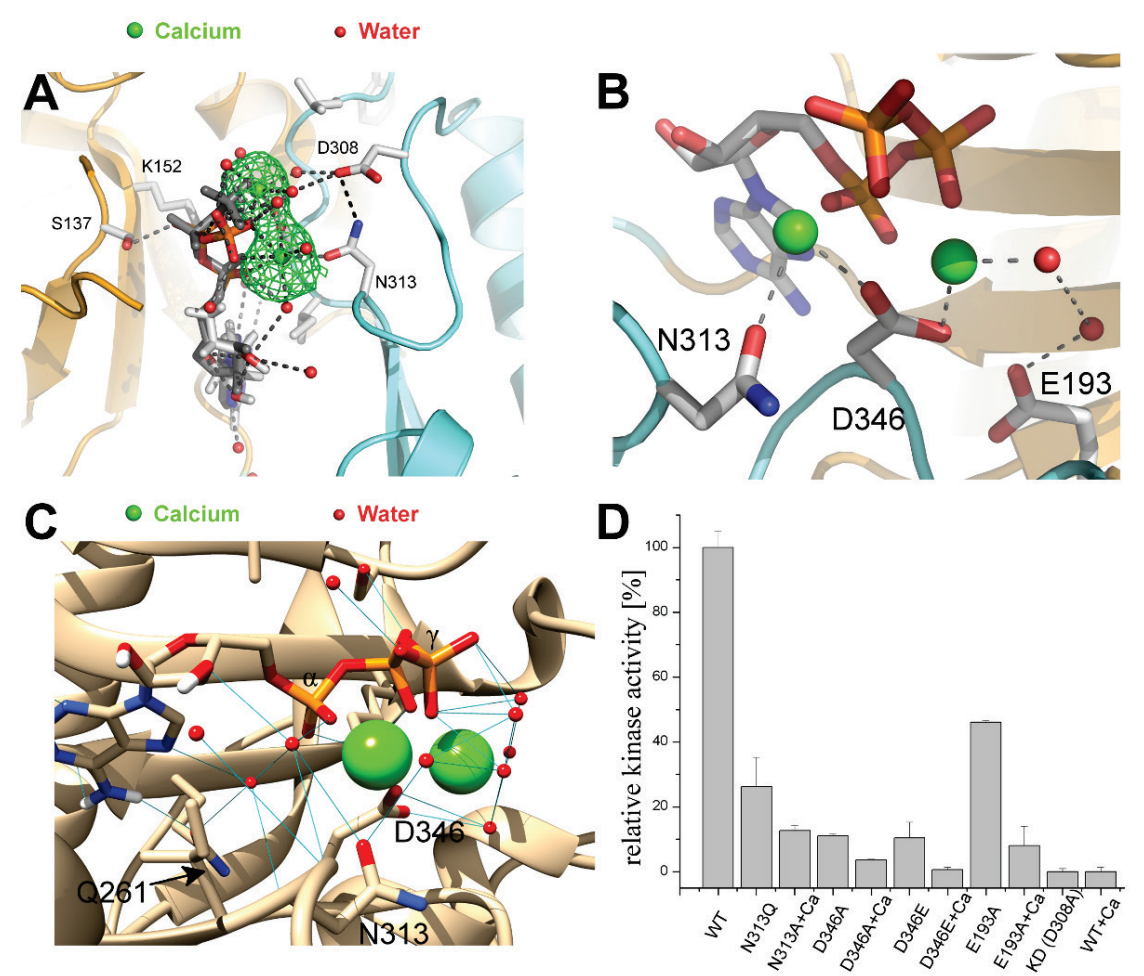

Fig. 3. Structural and functional analysis of PI4K IIa inhibition by calcium. A) The network of hydrogen bonds in the active site. C-lobe is colored in cyan, $\mathrm{N}$-lobe in orange. The ATP is colored according to its elements. Calcium is shown as green spheres, water molecules as red spheres. The unbiased Fo-Fc map at $3 \sigma$ is shown for the calcium atoms. B) Detailed view of the active site. Colored as previous panel. C) The complex water network that holds the two calcium atoms in place. D) Mutagenesis analysis of the active site. The activity of each mutant was measured with and without $10 \mathrm{mM}$ calcium ions and compared to the wt-enzyme.
We solved the structure of calcium bound PI4K II $\alpha$ to understand how calcium ions inhibit the enzyme at the atomic level. The structure revealed a complex network of hydrogen bonds formed between two calcium atoms, water molecules and residues within the active site (Fig. 3). We wanted to prepare a kinase that would not be inhibited by calcium because it would be a great tool to explore the physiological role of PI4K II $\alpha$ calcium inhibition. We selected three residues that directly (N313, D346) or indirectly (E193) bind calcium (Figs 3B and 3C) but are not essential for kinase activity for mutagenesis analysis (Fig. 3D). Based on our structure we hypothesized that some of the mutants (N313A, D346A, D346E, E193A) could retain high kinase activity but would not be inhibited by calcium. However, only E193A mutant retained significant kinase activity ( $46 \pm 2 \%$ ) but was still almost 
entirely inhibited by calcium $(8 \pm 5.3 \%)$ just as the wild type enzyme $(1 \pm 2.4 \%)$.

\section{Discussion}

The motivation for this study was to compare the active sites of the PI4K II $\alpha$ kinase with and without a ligand and to understand the PI4K II $\alpha$ inhibition at the atomic level. Our data suggest that the active site of PI4K II $\alpha$ is relatively rigid and ready for the nucleotide - no induced fit is required besides the ATP phosphate binding G-loop. We identified the G-loop and aspartate D346 (Figs $2 \mathrm{~A}$ and $2 \mathrm{~B}$ ) as the most flexible parts of the PI4K II $\alpha$ active site. D346 changes its conformation to form a hydrogen bond with the MD59 nucleotide analogue. Our data suggest D346 is the most flexible residue of the active site highlighting its importance for inhibitor design. It could be argued that conformational rigidity of the active site could be a crystal packing artifact. However, the active site is not directly involved in crystal packing and in the ADP bound structure published by Zhou and colleagues (2014) has the same conformation as in our previous ATP bound structure (Baumlova et al. 2014) despite different crystallization constructs and spacegroups. However, the conformation of the lateral C-lobe pocket is wholly crystal packing dependent. It has the same conformation in our previous ATP bound structure (Baumlova et al. 2014) and in the apo-state reported here (Fig. 2C) but it has a different conformation in the structure by Zhou and colleagues (2014) (different construct and spacegroup). The PI4K II $\alpha$ enzyme also contains a lateral pocket with surface exposed tryptophan residues that can be inserted into lipid bilayer, a process important for proper positioning of the enzyme (Zhou et al. 2014, Baumlova et al. 2014) that is also known to influence the biophysical properties of the lipid membrane (Hurley et al. 2010, Rozycki et al. 2012). However, our data suggest that the observed conformation of the lateral C-lobe pocket was governed by crystal contacts.

PI4K II $\alpha$ kinase activity is associated with synaptic vesicles (Guo et al. 2003) and synaptic vesicles are able to rapidly fuse in response to calcium (Jahn and Fasshauer 2012) implying possible role of calcium in regulation of PI4K II $\alpha$ activity in respect to the synaptic vesicles fusion process. We solved the structure of calcium bound PI4K II $\alpha$ to gain further knowledge of its architecture. We discovered that the ATP $\gamma$-phosphate has a different conformation when calcium is bound (Fig. 3). In fact, calcium atoms are held in place by a complex water network, extending from the calcium mainly to $\alpha$ - and $\gamma$-phosphates of the ATP molecule and to residues Asp346, Asn313, Gln261 and Glu193 (Figs 3B and 3C). We speculate that the two calcium atoms lock the ATP molecule and prevent its hydrolysis thus effectively inhibiting the enzyme. To understand the physiological role of calcium in the regulation of PI4K II $\alpha$ an active enzyme not inhibited by calcium is needed. We prepared several mutants based on our structure (N313A, D346A, D346E, E193A). Unfortunately, neither mutant had the desired properties.

To summarize, our data reveal details about the architecture of PI4K II $\alpha$ active site that should be helpful for future inhibitor design. PI4K II $\alpha$ is a known host factor for several intracellular bacteria such as Chlamydia trachomatis or Legionella pneumophila (Pizarro-Cerda et al. 2007, Pizarro-Cerda et al. 2012, Moorhead et al. 2010, Weber et al. 2006) and thus considered as a potential drug target. When computational algorithms search the chemical space for suitable compounds we propose that all the residues in the active should be fixed with the exception of the G-loop and aspartate D346.

\section{Conflict of Interest}

There is no conflict of interest.

\section{Acknowledgements}

Crystallographic data were collected at beamline 14-1 operated by the Helmholtz-Zentrum Berlin (HZB) at the BESSY II electron storage ring. This project was supported by Project InterBioMed LO1302 from Ministry of Education of the Czech Republic.

\section{Pdb accession code}

The coordinates have been deposited in PDB under ID code 5EUT (the apo-enzyme) and 5I0N (the calcium bound enzyme).

\section{References}

ADAMS PD, AFONINE PV, BUNKOCZI G, CHEN VB, DAVIS IW, ECHOLS N, HEADD JJ, HUNG LW, KAPRAL GJ, GROSSE-KUNSTLEVE RW, MCCOY AJ, MORIARTY NW, OEFFNER R, READ RJ, RICHARDSON DC, RICHARDSON JS, TERWILLIGER TC, ZWART PH: PHENIX: a comprehensive Python-based system for macromolecular structure solution. Acta Crystallogr D Biol Crystallogr 66: 213-221, 2010. 
ALTAN-BONNET N, BALLA T: Phosphatidylinositol 4-kinases: hostages harnessed to build panviral replication platforms. Trends Biochem Sci 37: 293-302, 2012.

BAUMLOVA A, CHALUPSKA D, ROZYCKI B, JOVIC M, WISNIEWSKI E, KLIMA M, DUBANKOVA A, KLOER DP, NENCKA R, BALLA T, BOURA E: The crystal structure of the phosphatidylinositol 4-kinase IIalpha. EMBO Rep 15: 1085-1092, 2014.

BOURA E, HURLEY JH: Structural basis for membrane targeting by the MVB12-associated beta-prism domain of the human ESCRT-I MVB12 subunit. Proc Natl Acad Sci USA 109: 1901-1906, 2012.

BOURA E, NENCKA R: Phosphatidylinositol 4-kinases: function, structure, and inhibition. Exp Cell Res 337: 136-145, 2015.

BOURA E, REZABKOVA L, BRYNDA J, OBSILOVA V, OBSIL T: Structure of the human FOXO4-DBD-DNA complex at 1.9 A resolution reveals new details of FOXO binding to the DNA. Acta Crystallogr D Biol Crystallogr 66: 1351-1357, 2010.

BROWN JR, AUGER KR: Phylogenomics of phosphoinositide lipid kinases: perspectives on the evolution of second messenger signaling and drug discovery. BMC Evol Biol 11: 4, 2011.

BURKE JE, INGLIS AJ, PERISIC O, MASSON GR, MCLAUGHLIN SH, RUTAGANIRA F, SHOKAT KM, WILLIAMS RL: Structures of PI4KIIIbeta complexes show simultaneous recruitment of Rab11 and its effectors. Science 344: 1035-1038, 2014.

CAUVIN C, ECHARD A: Phosphoinositides: lipids with informative heads and mastermind functions in cell division. Biochim Biophys Acta 1851: 832-843, 2015.

CLAYTON EL, MINOGUE S, WAUGH MG: Mammalian phosphatidylinositol 4-kinases as modulators of membrane trafficking and lipid signaling networks. Prog Lipid Res 52: 294-304, 2013.

DEJMEK M, SALA M, HREBABECKY H, DRACINSKY M, PROCHAZKOVA E, CHALUPSKA D, KLIMA M, PLACKOVA P, HAJEK M, ANDREI G, NAESENS L, LEYSSEN P, NEYTS J, BALZARINI J, BOURA E, NENCKA R: Norbornane-based nucleoside and nucleotide analogues locked in North conformation. Bioorg Med Chem 23: 184-191, 2015.

DORNAN GL, MCPHAIL JA, BURKE JE: Type III phosphatidylinositol 4 kinases: structure, function, regulation, signalling and involvement in disease. Biochem Soc Trans 44: 260-266, 2016.

DOWNING GJ, KIM S, NAKANISHI S, CATT KJ, BALLA T: Characterization of a soluble adrenal phosphatidylinositol 4-kinase reveals wortmannin sensitivity of type III phosphatidylinositol kinases. Biochemistry 35: 3587-3594, 1996.

EMSLEY P, LOHKAMP B, SCOTT WG, COWTAN K: Features and development of Coot. Acta Crystallogr D Biol Crystallogr 66: 486-501, 2010.

GORREC F: The MORPHEUS protein crystallization screen. J Appl Crystallogr 42: 1035-1042, 2009.

GUO J, WENK MR, PELLEGRINI L, ONOFRI F, BENFENATI F, DE CAMILLI P: Phosphatidylinositol 4-kinase type IIalpha is responsible for the phosphatidylinositol 4-kinase activity associated with synaptic vesicles. Proc Natl Acad Sci USA 100: 3995-4000, 2003.

HAMMOND GR, BALLA T: Polyphosphoinositide binding domains: key to inositol lipid biology. Biochim Biophys Acta 1851: 746-758, 2015.

HURLEY JH, BOURA E, CARLSON LA, ROZYCKI B: Membrane budding. Cell 143: 875-887, 2010.

JAHN R, FASSHAUER D: Molecular machines governing exocytosis of synaptic vesicles. Nature 490: 201-207, 2012.

JOVIC M, KEAN MJ, SZENTPETERY Z, POLEVOY G, GINGRAS AC, BRILL JA, BALLA T: Two phosphatidylinositol 4-kinases control lysosomal delivery of the Gaucher disease enzyme, betaglucocerebrosidase. Mol Biol Cell 23: 1533-1545, 2012.

JOVIC M, KEAN MJ, DUBANKOVA A, BOURA E, GINGRAS AC, BRILL JA, BALLA T: Endosomal sorting of VAMP3 is regulated by PI4K2A. J Cell Sci 127: 3745-3756, 2014.

KLIMA M, BAUMLOVA A, CHALUPSKA D, HREBABECKY H, DEJMEK M, NENCKA R, BOURA E: The highresolution crystal structure of phosphatidylinositol 4-kinase IIbeta and the crystal structure of phosphatidylinositol 4-kinase IIalpha containing a nucleoside analogue provide a structural basis for isoformspecific inhibitor design. Acta Crystallogr D Biol Crystallogr 71: 1555-1563, 2015. 
KLIMA M, TOTH DJ, HEXNEROVA R, BAUMLOVA A, CHALUPSKA D, TYKVART J, REZABKOVA L, SENGUPTA N, MAN P, DUBANKOVA A, HUMPOLICKOVA J, NENCKA R, VEVERKA V, BALLA T, BOURA E: Structural insights and in vitro reconstitution of membrane targeting and activation of human PI4KB by the ACBD3 protein. Sci Rep 6: 23641, 2016.

KRUG M, WEISS MS, HEINEMANN U, MUELLER U: XDSAPP: a graphical user interface for the convenient processing of diffraction data using XDS. J Appl Crystallogr 45: 568-572, 2012.

MEJDROVA I, CHALUPSKA D, KOGLER M, SALA M, PLACKOVA P, BAUMLOVA A, HREBABECKY H, PROCHAZKOVA E, DEJMEK M, GUILLON R, STRUNIN D, WEBER J, LEE G, BIRKUS G, MERTLIKOVA-KAISEROVA H, BOURA E, NENCKA R: Highly selective phosphatidylinositol 4-kinase iiibeta inhibitors and structural insight into their mode of action. J Med Chem 58: 3767-3793, 2015.

MOORHEAD AM, JUNG JY, SMIRNOV A, KAUFER S, SCIDMORE MA: Multiple host proteins that function in phosphatidylinositol-4-phosphate metabolism are recruited to the chlamydial inclusion. Infect Immun $\mathbf{7 8}$ : 1990-2007, 2010.

MUELLER U, DAROWSKI N, FUCHS MR, FORSTER R, HELLMIG M, PAITHANKAR KS, PUHRINGER S, STEFFIEN M, ZOCHER G, WEISS MS: Facilities for macromolecular crystallography at the HelmholtzZentrum Berlin. J Synchrotron Radiat 19: 442-449, 2012.

NEMECEK D, BOURA E, WU W, CHENG N, PLEVKA P, QIAO J, MINDICH L, HEYMANN JB, HURLEY JH, STEVEN AC: Subunit folds and maturation pathway of a dsRNA virus capsid. Structure 21: 1374-1383, 2013.

PIZARRO-CERDA J, PAYRASTRE B, WANG YJ, VEIGA E, YIN HL, COSSART P: Type II phosphatidylinositol 4-kinases promote Listeria monocytogenes entry into target cells. Cell Microbiol 9: 2381-2390, 2007.

PIZARRO-CERDA J, KUHBACHER A, COSSART P: Entry of Listeria monocytogenes in mammalian epithelial cells: an updated view. Cold Spring Harb Perspect Med 2: a010009, 2012.

ROZYCKI B, BOURA E: Large, dynamic, multi-protein complexes: a challenge for structural biology. J Phys Condens Matter 26: 463103, 2014.

ROZYCKI B, BOURA E, HURLEY JH, HUMMER G: Membrane-elasticity model of Coatless vesicle budding induced by ESCRT complexes. PLoS Comput Biol 8: e1002736, 2012.

TAI AW, BOJJIREDDY N, BALLA T: A homogeneous and nonisotopic assay for phosphatidylinositol 4-kinases. Anal Biochem 417: 97-102, 2011.

TAN J, BRILL JA: Cinderella story: PI4P goes from precursor to key signaling molecule. Crit Rev Biochem Mol Biol 49: 33-58, 2014.

WAUGH MG: PIPs in neurological diseases. Biochim Biophys Acta 1851: 1066-1082, 2015.

WEBER SS, RAGAZ C, REUS K, NYFELER Y, HILBI H: Legionella pneumophila exploits PI(4)P to anchor secreted effector proteins to the replicative vacuole. PLoS Pathog 2: e46, 2006.

ZHOU Q, LI J, YU H, ZHAI Y, GAO Z, LIU Y, PANG X, ZHANG L, SCHULTEN K, SUN F, CHEN C: Molecular insights into the membrane-associated phosphatidylinositol 4-kinase IIalpha. Nat Commun 5: 3552, 2014. 